\title{
High-precision pose and velocity measuring method for projectiles based on kalman filtering algorithm
}

\author{
Liang Zhang ${ }^{1}$, Lizhi Qian ${ }^{1}$, Quanli Ning ${ }^{1}$, Jingxiao Wang ${ }^{1}$ \\ (1. New Star Research Institude of Applied Technology, Hefei, China)
}

Keywords: projectiles; kalman filtering algorithm; trajectory; tracking

\begin{abstract}
An original method is presented in this paper, which attempts to make use of the kalman filtering algorithm and maintain accuracy in position, attitude and velocity estimation for a fast moving projectile, the velocity and the pose (position and attitude) could also be obtained, then the simulation are presented and compared with the conventional least square method. The simulation result showed that the kalman filter algorithm is more accurate and stability than the conventional least squares algorithm, meanwhile, the trajectory of the projectile obtained by the kalman filter algorithm is very close to the real trajectory.
\end{abstract}

\section{Introduction}

It is necessary to capture the motion parameters of fast moving projectiles, such as pose (including position and attitude), velocity (including speed and moving direction), and angle of attack (AOA, the angle of the attitude and the moving direction). These parameters have major influence on the weapon performance and accuracy. Until recently, many optoelectronic-based [12], image-based methods [3-6], etc., have been employed to perform this task. Because of the relatively high precision signal that the radar systems could provide, and maturity of the signal processing algorithms, radar tracking methods have many benefits against alternative methods especially the target is a fast moving[7-9]. Therefore, it is a trend to develop a new method for projectile tracking such as high-precision pose and velocity measuring.

An original method is presented in this paper, which attempts to make use of the kalman filtering algorithm and maintain accuracy in position, attitude and velocity estimation for a fast moving projectile, the velocity and the pose (position and attitude) could also be obtained, then the simulation are presented and compared with the conventional least square method.

\section{Measuring method for projectiles tracking}

In order to capture the motion parameters of fast moving projectiles, it can be divided into three steps.

Firstly, a system of gathering data about where the projectile is located should be provided. The system discussed in this paper is radar. Secondly, a method for accurate estimation of the projectile's position, velocity should be presented. The method used in this paper is kalman filtering algorithm for projectiles tracking, finally, once the enough data can be collected and analyzed, the projectile trajectory can be determined and simulated using the computer

\section{Target detection}

The purpose of this step is to take raw environmental data and turn it into a series of points plotted in Cartesian space. These points represent the trajectory of the projectile and are made up of a list of points $(\mathrm{x} 0, \mathrm{y} 0, \mathrm{z} 0) . .(\mathrm{xn}, \mathrm{yn}, \mathrm{zn})$ where $\mathrm{n}$ is the number of positions sampled and a time difference $t$ which is the time difference between each sample[10].

For tracking objects at both long and short distances, Tracking radar systems are used to measure the target's relative position in range, azimuth angle, elevation angle, and velocity. Then, by using and keeping track of these measured parameters the radar can predict their future values. Target tracking is important to military radars as well as to most civilian radars. In military radars, tracking 
is responsible for fire control and missile guidance; in fact, missile guidance is almost impossible without proper target tracking. Tracking techniques can be divided into range/velocity tracking and angle tracking.

\section{A. Angle Tracking}

Angle tracking is concerned with generating continuous measurements of the target's angular position in the azimuth and elevation coordinates. The accuracy of early generation angle tracking radars depended heavily on the size of the pencil beam employed. Most modern radar systems achieve very fine angular measurements by utilizing monopulse tracking techniques.

Tracking radars use the angular deviation from the antenna main axis of the target within the beam to generate an error signal. This deviation is normally measured from the antenna's main axis. The resultant error signal describes how much the target has deviated from the beam main axis. Then, the beam position is continuously changed in an attempt to produce a zero error signal. If the radar beam is normal to the target (maximum gain), then the target angular position would be the same as that of the beam. In practice, this is rarely the case.

In order to be able to quickly achieve changing the beam position, the error signal needs to be a linear function of the deviation angle. It can be shown that this condition requires the beam's axis to be squinted by some angle (squint angle) off the antenna's main axis.

\section{B. Amplitude Comparison Monopulse}

Amplitude comparison monopulse tracking is similar to lobing in the sense that four squinted beams are required to measure the target's angular position. The difference is that the four beams are generated simultaneously rather than sequentially. For this purpose, a special antenna feed is utilized such that the four beams are produced using a single pulse, hence the name "monopulse."Additionally, monopulse tracking is more accurate and is not susceptible to lobing anomalies, such as AM jamming and gain inversion ECM. Finally, in sequential and conical lobing variations in the radar echoes degrade the tracking accuracy; however, this is not a problem for monopulse techniques since a single pulse is used to produce the error signals. Monopulse tracking radars can employ both antenna reflectors as well as phased array antennas.

Fig. 1 show a typical monopulse antenna pattern. The four beams A, B, C, and D represent the four conical scan beam positions. Four feeds, mainly horns, are used to produce the monopulse antenna pattern. Amplitude monopulse processing requires that the four signals have the same phase and different amplitudes.

The radar continuously compares the amplitudes and phases of all beam returns to sense the amount of target displacement off the tracking axis. It is critical that the phases of the four signals be constant in both transmit and receive modes. For this purpose, either digital networks or microwave comparator circuitry are utilized. Fig. 2 shows a block diagram for a typical micro-wave comparator, where the three receiver channels are declared as the sum channel, elevation angle difference channel, and azimuth angle difference channel.

To generate the elevation difference beam, one can use the beam difference $(A-D)$ or $(B-C)$. However, by first forming the sum patterns $(A+B)$ and $(D+C)$ and then computing the difference $(A+B)-(D+C)$, we achieve a stronger elevation difference signal, $\Delta_{e t}$, Similarly, by first forming the sum patterns $(A+D)$ and $(B+C)$ and then computing the difference $(A+D)-(B+C)$, a stronger azimuth difference signal, $\Delta_{a z}$, is produced.

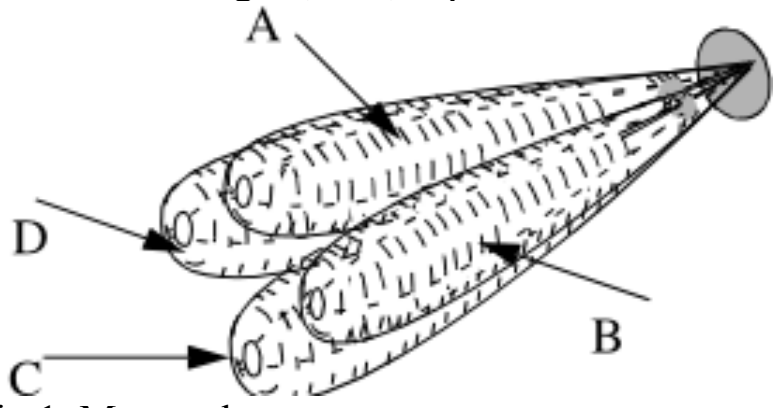

Fig.1. Monopulse antenna pattern

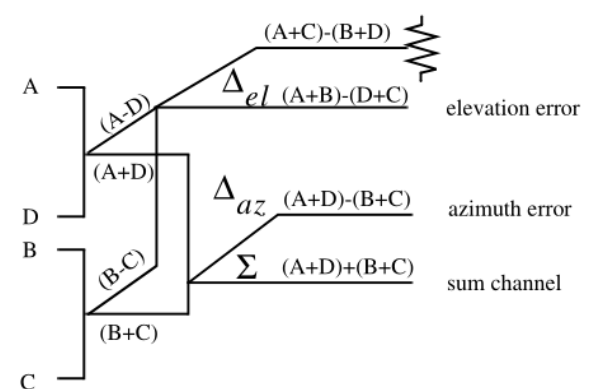

Fig 2. Monopulse comparator 


\section{Phase Comparison Monopulse}

Phase comparison monopulse is similar to amplitude comparison monopulse in the sense that the target angular coordinates are extracted from one sum and two difference channels. The main difference is that the four signals produced

in amplitude comparison monopulse will have similar phases but different amplitudes; however, in phase comparison monopulse the signals have the same amplitude and different phases. Phase comparison monopulse tracking radars use a minimum of a two-element array antenna for each coordinate (azimuth and elevation), as illustrated in Fig.3. A phase error signal (for each coordinate) is computed from the phase difference between the signals generated in the antenna elements.

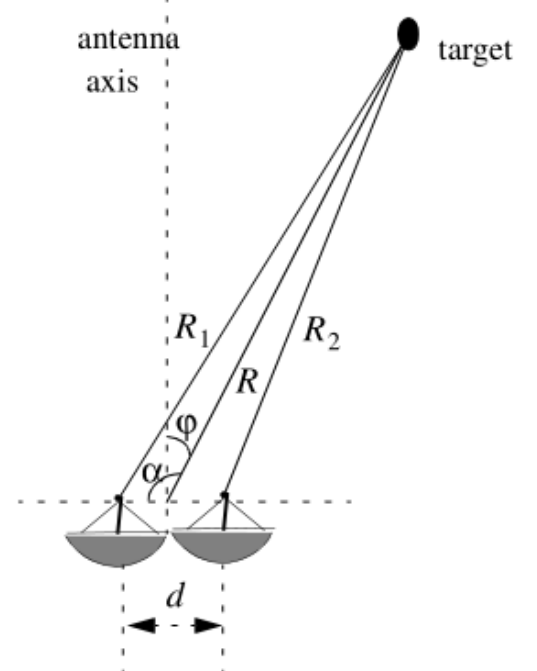

Fig.3. Single coordinate phase comparison monopulse antenna

Consider Fig. 3 ; since the angle $\alpha$ is equal to $\varphi+\pi / 2$, it follows that

$$
\begin{aligned}
& R_{1}^{2}=R^{2}+\left(\frac{d}{2}\right)^{2}-2 \frac{d}{2} R \cos \left(\varphi+\frac{\pi}{2}\right) \\
& =R^{2}+\frac{d^{2}}{4}-d R \sin \varphi
\end{aligned}
$$

and since $d<<R$ we can use the binomial series expansion to get

Similarly,

$$
R_{1} \approx R\left(1+\frac{d}{2 R} \sin \varphi\right)
$$

$$
R_{2} \approx R\left(1+\frac{d}{2 R} \sin \varphi\right)
$$

The phase difference between the two elements is then given by

$$
\varphi=\frac{2 \pi}{\lambda}\left(R_{1}-R_{2}\right)=\frac{2 \pi}{\lambda} d \sin \varphi
$$

where is the wavelength. The phase difference $\varphi$ is used to determine the angular target location. Note that if $\phi=0$, then the target would be on the antenna's main axis. The problem with this phase comparison monopulse technique is that it is quite difficult to maintain a stable measurement of the off boresight angle $\varphi$, which causes serious performance degradation. This problem can be overcome by implementing a phase comparison monopulse system as illustrated in Fig.4.

The (single coordinate) sum and difference signals are, respectively, given by

$$
\begin{aligned}
& \sum(\varphi)=S_{1}+S_{2} \\
& \Delta \varphi=S_{1}-S_{2}
\end{aligned}
$$


where the and are the signals in the two elements. Now, since $S_{1}$ and $S_{2}$ have similar amplitude and are different in phase by $\phi$, we can write

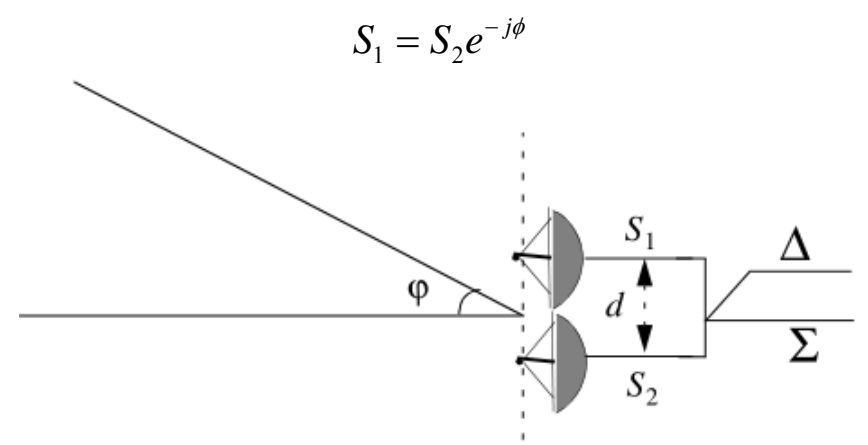

Fig.4. Single coordinate phase monopulse antenna with sum and difference channels It follows that

$$
\begin{aligned}
& \Delta(\varphi)=S_{2}\left(1-e^{-j \varphi}\right) \\
& \sum(\varphi)=S_{2}\left(1+e^{-j \varphi}\right)
\end{aligned}
$$

The phase error signal is computed from the ratio $\Delta / \Sigma$. More precisely,

$$
\frac{\Delta}{\Sigma}=\frac{1-e^{-j \phi}}{1+e^{-j \phi}}=j \tan \left(\frac{\phi}{2}\right)
$$

which is purely imaginary. The modulus of the error signal is then given by

$$
\frac{|\Delta|}{|\Sigma|}=\tan \left(\frac{\phi}{2}\right)
$$

This kind of phase comparison monopulse tracker is often called the half-angle tracker.

\section{Range Tracking}

Target range is measured by estimating the round-trip delay of the transmitted pulses. The process of continuously estimating the range of a moving target is known as range tracking. Since the range to a moving target is changing with time, the range tracker must be constantly adjusted to keep the target locked in range. This can be accomplished using a split gate system, where two range gates (early and late) are utilized. The concept of split gate tracking is illustrated in Fig.5, where a sketch of a typical pulsed radar echo is shown in the figure. The early gate opens at the anticipated starting time of the radar echo and lasts for half its duration. The late gate opens at the center and closes at the end of the echo signal. For this purpose, good estimates of the echo duration and the pulse centertime must be reported to the range tracker so that the early and late gates can be placed properly at the start and center times of the expected echo. This reporting process is widely known as the "designation process."

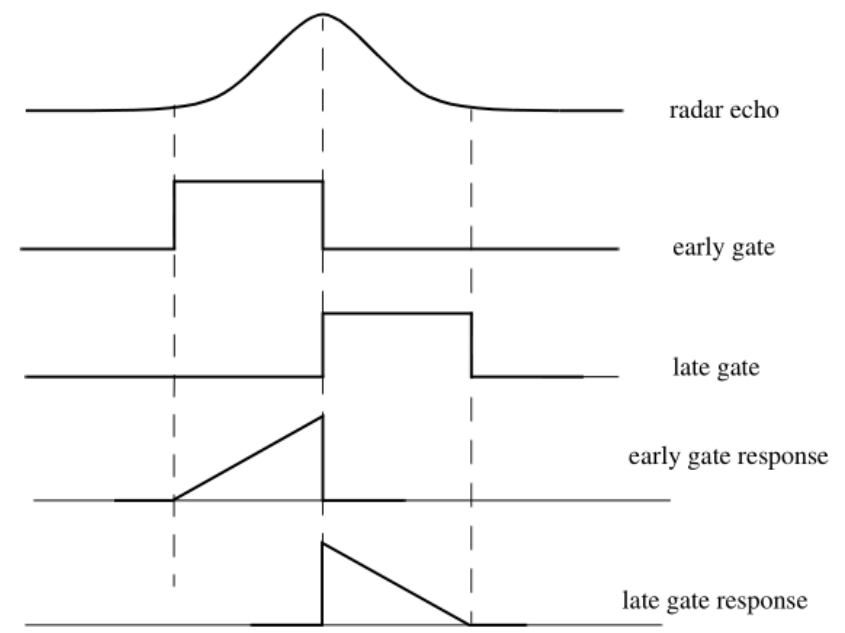

Fig 5. Illustration of split-range gate 
The early gate produces positive voltage output while the late gate produces negative voltage output. The outputs of the early and late gates are subtracted, and the difference signal is fed into an integrator to generate an error signal. If both gates are placed properly in time, the integrator output will be equal to zero. Alternatively, when the gates are not timed properly, the integrator output is not zero, which gives an indication that the gates must be moved in time, left or right depending on the sign of the integrator output.

\section{Accurate target positioning}

Once a projectile has been detected, it needs to be tracked to determine its trajectory. The kalman filtering algorithm is used to estimation the accurate position and velocity of the fast moving projectile[11].

\section{A. the principle of the Kalman filter alogrithm}

Kalman filter is an optimal Recursive Data Processing Algorithm. It consists of the following two phases- (i) prediction and (ii) correction. The first refers to the prediction of the next state using the current set of observations and update the current set of predicted measurements. The second updates the predicted values and gives a much better approximation of the next state. It attempts to achieve a balance between predicted values and noisy measurements. The values of the weights are determined by modeling the state equations.

Kalman filter algorithm can be given as

$$
P_{k}=\text { A.P. } A^{-1}+Q
$$

Kalman filter working depends on Kalman update given by

$$
\begin{gathered}
K_{k}=P_{k}^{-1} \cdot H^{T}\left(H \cdot P_{K}^{-1} \cdot H^{T}+R\right)^{-1} \\
x=x_{0}+K_{k}\left(\left[x_{c}, y_{c}\right]^{T}-H \cdot x_{0}\right)
\end{gathered}
$$

Where: $x_{c}, y_{c}$-centre coordinates of the ball, $R$-measurement noise covariance, $K_{k}$-Kalman update, ${ }^{x_{0}}$-initial estimation of the ball.

From the above equation, it is clear that the kalman filter gives more preference to the measurement values, if they are trusted to be nearer to the actual values or else, to the estimated values.

$$
\begin{gathered}
P_{k+1}=1-K_{k} \cdot H \cdot P_{k} \\
R=E\left(v_{k} v_{k}\right)^{T}
\end{gathered}
$$

$V_{k}$-standard deviation of measurement noise.

These equations clearly indicate that the kalman gain depends on process noise covariance(R). This kalman gain, in turn alters the predicted position. Hence initialization of the kalman filter is an important task for a kalman filter designer. The flow chart of kalman filter alogrtihm is shown as Fig.6.

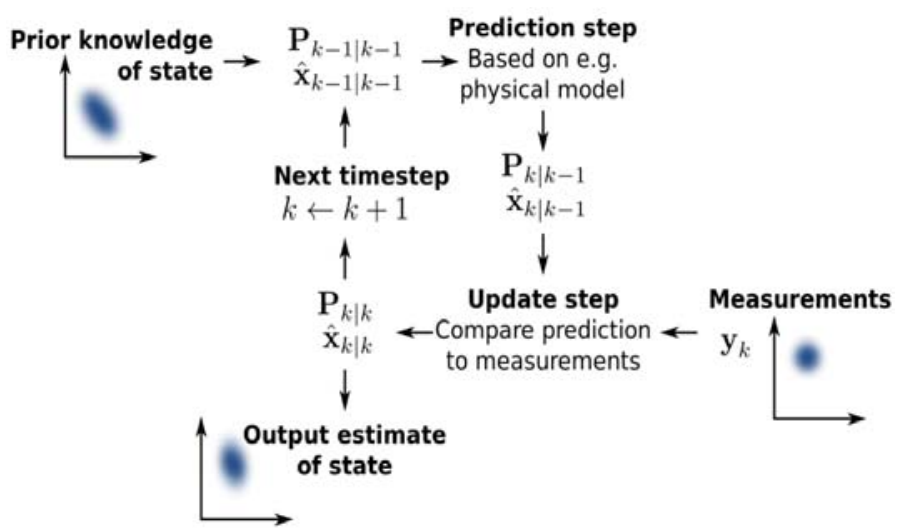

Fig.6 The flow chart of kalman filter alogrtihm 
From the above equation, it is clear that the kalman filter gives more preference to the measurement values, if they are trusted to be nearer to the actual values or else, to the estimated values

\section{B. The process of the tracking based on kalman filter alogrtihm}

In data processing, it requires the use of the limited observation time to collect observations to estimate the state of linear discrete-time dynamic systems. Assume that the system model equation is that:

$$
S_{k+1}=\Phi_{k} \hat{S}_{k}+B_{k} u_{k}+G_{k} v_{k}
$$

Where: $S_{k}$-n dimensional vector of system status in $k$ time

$\Phi_{k}-n \times n$ order state transition matrix in $k$ time

$u_{k}$-the $n$ dimension of the input vector

$B_{k}$-the $n \times p$ order of the input matrix

$\mathrm{v}_{\mathrm{k}}-q$ dimensional random vector, a Gaussian white noise distribution;

$G_{k^{-}} n \times q$ dimensional real-valued matrix, that is:

$$
\begin{gathered}
E\left\{v_{k}\right\}=0 \\
E\left\{G_{k} v_{k} v_{j}^{T} G_{j}^{T}\right\}=Q_{k} \delta_{k j}
\end{gathered}
$$

Observation equation is a linear function, namely

$$
z_{k}=H_{k} S_{k}+L_{k} w_{k}
$$

Where: $Z_{k}-m$ dimension observation vector in $k$ time;

$H_{k}-m \times n$ order of the observation matrix;

$w_{k}-m$ dimensional measurement noise, Gaussian white noise distribution; that is

$$
\begin{gathered}
E\left\{w_{k}\right\}=0 \\
E\left\{L_{k} w_{k} w_{j}^{T} L_{j}^{T}\right\}=R_{k} \delta_{k j}
\end{gathered}
$$

Assume that vk and wk is independent of each other, namely

$$
E\left\{v_{k} w_{k}^{T}\right\}=0
$$

Utilizing the results of the MMSE estimates are derived by the system state prediction equation of state filter equations, filter gain equation, the residual covariance matrix, usually called Kalman filter equations. Kalman filter equations can be derived based on the assumption of linear model and the Gaussian distribution, application guidelines to obtain the best estimate of the optimum filter to be the distribution function of the process and not make any assumptions using linear least square estimate.

Assume $\hat{S}_{k / k}$ is the least mean-square estimate of the sk before and after $\mathrm{k}$ time, that is:

$$
\hat{S}_{k / k}=E\left\{s_{k} / z^{k}\right\}
$$

Where $z^{k}=\left(z_{0}, z_{1}, \cdots, z_{k}\right)$

The corresponding covariance matrix is shown as:

$\hat{P}_{k / k}=E\left\{\left(s_{k}-\hat{s}_{k / k}\right) \cdot\left(s_{k}-\hat{s}_{k / k}\right)^{T} / z^{k}\right\}=E\left\{\Delta s_{k} \cdot \Delta s_{k}^{T} / z^{k}\right\}$ Where: $\Delta s_{k}=s_{k}-\hat{s}_{k / k}$

It is known $\hat{P}_{k / k}$ as k time filter covariance matrix, the iteration relations of the state predicted value in $\mathrm{k}+1$ time, the covariance matrix and the filter value in $\mathrm{k}$ time and covariance matrix is established, the state predicted value in $\mathrm{k}+1$ time $\hat{S}_{k+1 / k}$ is exported as:

Its corresponding error is:

$$
\hat{s}_{k+1 / k}=\Phi_{k} s_{k / k}+B_{k} u_{k}
$$

$$
\Delta s_{k+1 / k}=s_{k+1 / k}-\hat{s}_{k+1 / k}=\Phi_{k} \Delta s_{k / k}+G_{k} v_{k}
$$

The prediction covariance matrix in $\mathrm{k}+1$ time is show as: 


$$
\hat{P}_{k+1 / k}=E\left\{\Delta s_{k+1 / k} \cdot \Delta s_{k+1}^{T} / z^{k}\right\}=\Phi_{k} P_{k / k} \Phi_{k}^{T}+Q_{k}
$$

The residuals and covariance matrix is deduced as follow, Set $\hat{Z}_{k+1 / k}$ as the minimum mean square error estimation between $z^{k}$ and $z^{k+1}$ namely:

$$
\hat{Z}_{k+1}=E\left\{z_{k+1} / z^{k}\right\}
$$

Observation prediction model can be obtained as follows:

$$
\hat{Z}_{k+1}=H_{k+1} S_{k+1 / k}
$$

According to the residual definition as:

$$
v_{k+1}=z_{k+1}-\hat{Z}_{k+1 / k}=H_{k+1} \Delta s_{k+1 / k}+L_{k} w_{k+1}
$$

Covariance matrix of the residuals can be obtained as follows:

$$
\theta_{k+1}=E\left\{v_{k+1} \cdot v_{k+1}^{T} / z^{k}\right\}=H_{k+1} \hat{P}_{k+1 / k} H_{k+1}^{T}+R_{k+1}
$$

Assume $k+1$ time and previous observed value $z^{k+1}$ is obtained, the corresponding filter values $\hat{S}_{k+1 / k+1}$ and covariance matrix $P_{k+1 / k+1}$. can be exported. As the definition of the minimum estimate of covariance $\hat{S}_{k+1 / k+1}$, that is:

$$
\hat{s}_{k+1 / k+1}=E\left\{s_{k+1} / z^{k+1}\right\}=E\left\{s_{k+1} /\left(z^{k}, z_{k+1}\right)\right\}
$$

Using the minimum mean square estimate to obtain:

$$
\hat{x}=\bar{x}+P_{x z} P_{z z}^{-1}(z-\bar{z})
$$

$$
\begin{gathered}
P=P_{x x}-P_{x z} P_{z z}^{-1} P_{x z}^{T} \\
P_{x x}=E\left\{\Delta s_{k+1 / k} \cdot \Delta s_{k+1 / k}^{T} / z^{k}\right\}=P_{k+1 / k} \\
P_{x z}=E\left\{\Delta s_{k+1 / k} \cdot v_{k+1}^{T} / z^{k}\right\}=P_{k+1 / k} H_{k+1}^{T} \\
P_{z z}=E\left\{v_{k+1} \cdot v_{k+1}^{T} / z^{k}\right\}=\theta_{k+1}
\end{gathered}
$$

Filter recursive formula for the $k+1$ time available is shown as:

$$
\begin{aligned}
\hat{S}_{k+1 / k+1}= & \left.\hat{S}_{k+1 / k}+P_{k+1 / k} H_{k+1}^{T} \theta_{k+1}^{-1}\left(Z_{k+1}-\hat{Z}_{k+1 / k}\right)\right) \\
= & \hat{S}_{k+1 / k}+K_{k+1}\left(z_{k+1}-Z_{k+1 / k}\right)
\end{aligned}
$$

The filter gain matrix $K_{k+1}$ is defined as Kalman gain, that is:

$$
K_{k+1}=\hat{P}_{k+1 / k} H_{k+1}^{T} \theta_{k+1}^{-1}=\hat{P}_{k+1 / k} H_{k+1}^{T}\left(H_{k+1} \hat{P}_{k+1 / k} H_{k+1}^{T}+R_{k+1}\right)^{-1}
$$

It can be seen that the filter value in $k+1$ time is the predicted value in $\mathrm{k}+1$ time adding the correction value for the observation in $\mathrm{k}+1$ time, the weight effect is $K_{k+1}$. The covariance matrix filter is shown as:

$\hat{P}_{k+1 / k+1}=\hat{P}_{k+1 / k}+\hat{P}_{k+1 / k} H_{k+1}^{T} \theta_{k+1}^{-1} H_{k+1} \hat{P}_{k+1}=\left(1-K_{k+1} H_{k+1}\right) \hat{P}_{k+1 / k}$

This article intends to adopt the following steps to complete the tracking filter method:

(1) According to the observed and calculated values $\mathrm{Z1}$ and $\mathrm{Z} 2$ of the two previous positions, the initial value of the Kalman filter is obtained as:

$$
\hat{S}_{2 / 2}^{T}=\left[z_{2},\left(z_{2}-z_{1}\right) / T\right]
$$

(2) Assume the observation noise $\mathrm{w}$ is a stationary noise variance and zero-mean Gaussian distributed random variables, the process noise is independent of the initial conditions. The corresponding covariance matrix is exported as the following form

$$
P_{2 / 2}=\left[\begin{array}{cc}
\sigma_{w}^{2} & \sigma_{w} / T^{2} \\
\sigma_{w} / T^{2} & 2 \sigma_{w}^{2} / T^{2}
\end{array}\right]
$$

The formula of the estimated value of the Kalman filter is given as follows:

By filtering covariance matrix initial value to calculate the predicted covariance matrix, 
namely:

$$
\hat{P}_{k+1 / k}=\Phi \hat{P}_{k / k} \Phi^{T}+Q_{k}
$$

The estimated covariance matrix can be calculated as

$$
K_{k+1}=\hat{P}_{k+1 / k} H^{T}\left(H \hat{P}_{k+1 / k} H^{T}+R_{k+1}\right)^{-1}
$$

Filter covariance can be obtained as:

$$
\hat{P}_{k+1 / k+1}=\left(I-K_{k+1} H\right) \hat{P}_{k+1 / k}
$$

By the state of the predicted value, and the Kalman gain observed values, we can calculate the value of Kalman Filter:

$$
\hat{S}_{k+1 / k+1}=\hat{S}_{k+1 / k}+K_{k+1}\left(z_{k+1}-H_{k+1} \hat{S}_{k+1 / k}\right)
$$

\section{Simulation and analysis}

In this section, the performance of kalman filter algorithm is compared with the conventional least squares algorithm. The real trajectory of the projectile is also provided to verify the kalman filter algorithm. All the simulations are performed at one platform under the same environment.

The trajectory of the projectile using by the kalman filter algorithm and the conventional least squares algorithm is shown in Fig6, The real trajectory of the projectile is also plotted in the Fig.7. It can be seen that the kalman filter algorithm is more accurate than the conventional least squares algorithm, The trajectory of the projectile obtained by the kalman filter algorithm is very close to the real trajectory.

The direction error and the pitch error of the kalman filter algorithm and the conventional least square algorithm are shown as Fig.8 and Fig.9. It can be found that the direction error and the pitch error of the kalman filter algorithm are much less than the conventional least squares algorithm, From Fig.8 and Fig.9, It also can be seen that the kalman filter algorithm is more stability than the conventional least squares algorithm.

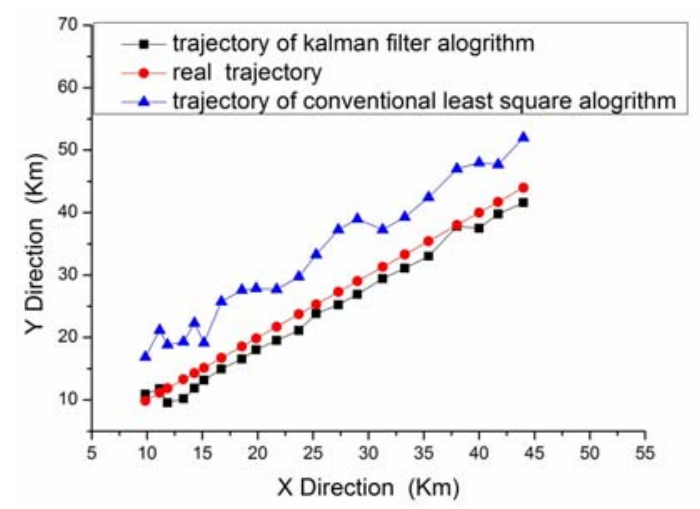

Fig.7 Trajectory of both algorithms

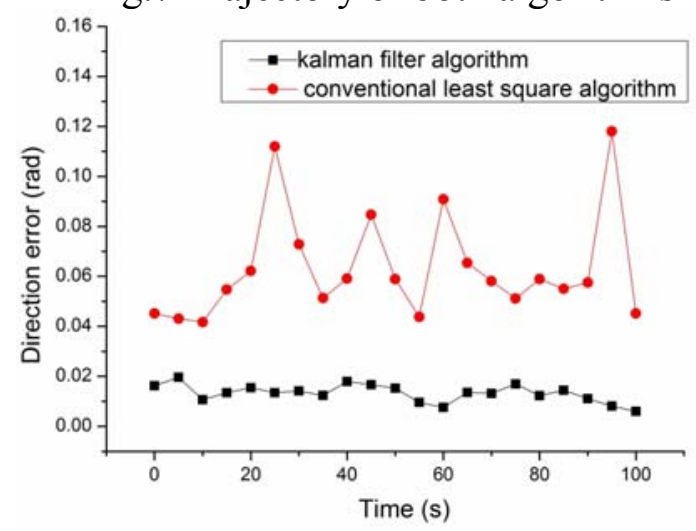

Fig.8 Direction error of both algorithms 


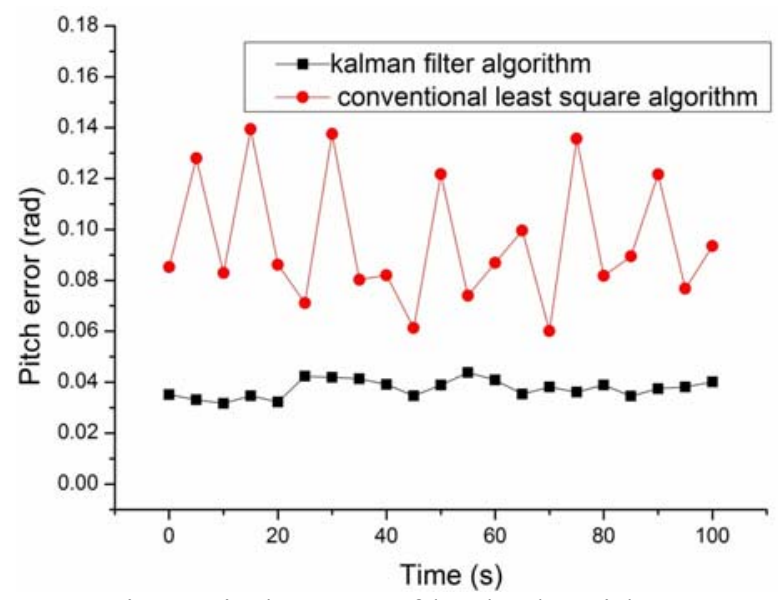

Fig.9 Pitch error of both algorithms

\section{Conclusion}

An original method is presented in this paper, which attempts to make use of the kalman filtering algorithm and maintain accuracy in position, attitude and velocity estimation for a fast moving projectile, the velocity and the pose (position and attitude) could also be obtained, then the simulation are presented and compared with the conventional least square method. The simulation result showed that the kalman filter algorithm is more accurate and stability than the conventional least squares algorithm, meanwhile the trajectory of the projectile obtained by the kalman filter algorithm is very close to the real trajectory.

\section{Acknowledgement}

This paper is supported by Equipment Development Fun Project: 9140A05020114JB91064

\section{References}

[1] Shan Chang-sheng. Measuring error analysis of projectile's yaw angle.J Journal of Ballistics 1995; 7(2):67-73

[2] Wang Miao, Li Hua. Research about capturing Of CCD vertical target measurement system. J Acta Photonica Sinica 2009, 38(6):1539-1542

[3] Liu Tong xian, Song Wei dong, Song Pi ji, et al. The Research on the Measurement of the Attack Angle of Flying Projectile by Linear Array CCD. J Optoelecfronic Technology, 2002, 22(4):228-230

[4] D. Jang, H. Choi. Active models for tracking moving objects. Pattern Recogn, 2000(33):11351146

[5] JIMFRON TAN, NICHOLAS KYFUAKOPOULOS. Implementation of a Tracking Kalman Filter on a Digital Signal Processor. IEEE TRANSACTIONS ON INDUSTRIAL ELECTRONICS, 1988, 35(1):126- 129

[6] Xin Li, Kejun Wang,Wei Wang and Yang Li A Multiple Object Tracking Method Using Kalman Filter. Proceedings of the 2010 IEEE International Conference on Information and Automation, Harbin, China, 2010

[7] Saira Saleem Pathan, Ayoub Al-Hamadi, Bernd Michaelis. Intelligent feature-guided multiobject tracking using Kalman filter. $2^{\text {nd }}$ International Conference on Computer Control and Communication, 2009

[8] X. Yu, H. W. Leong, C. Xu, and Q. Tian. Trajectory-based ball detection and tracking of broadcast soccer video. IEEE Transactions on Multimedia, 2006(8): 1164-1178 
[9] Jong-Yun Kim ; Tae-Yong Kim. Soccer Ball Tracking using Dynamic Kalman Filter with Velocity Control Computer Graphics. Sixth International Conference on Imaging and Visualization, 2009(8): 367-374

[10] Sinha A, Kirubarajan T, Bar-Shalom, Y,. Application of the Kalman-Levy Filter for Tracking Maneuvering Targets. IEEE Transactions on Aerospace and Electronic Systems, 2007(7): $1099-1107$

[11] N. Cui, L. Hong, J.R. Layne, A comparison of nonlinear filtering approaches with application to ground target tracking. Signal Processing, 2005, 85(8):1469-1492 